In another rare instance (Fig. 4), the sectoral radicle has attained direct nodal fusion with the median radicle (12) and has thus been drawn definitely into the mediosectoral linkage group. If the sectoral radicle faded out of existence or became merged with the arculus, the result would be that shown in Fig. 2.

In conclusion it may be remarked that while these phases are consecutive in form, they are contemporary in incidence, occurring as individual variations.

Mille Isles,

Arthur Willey,

St. Jérome,

Quebec.

May 25.

\section{The Neutrino Theory of Light}

Some time ago I directed attention ${ }^{2}$ to some serious difficulties connected with the neutrino theory of light as developed by Jordan and Kronig, namely, (1) the contradiction with the superposition principle especially in the three-dimensional case, and (2) the impossibility of expressing the Bose amplitudes $b(\nu)$ in terms of Fermi amplitudes $\gamma(v)$. This criticism has met with objections ${ }^{2,3}$. It has been pointed out that in Jordan's theory the introduction of neutrinoholes is essential and that operators satisfying the Bose commutation rules can be constructed if Dirac's theory of holes is used.

My present purpose is to elucidate some points connected with the operators considered, and to point out that (1) the problem of constructing Bose amplitudes remains unsolved, and (2) new difficulties arise in the theory when the neutrino-holes are introduced.

In a theory working with an indefinite number of particles, the following boundary condition for the wave functional is necessary. The probability of finding more than $N$ physical particles must tend to zero if $N \rightarrow \infty$. The boundary conditions in the ordinary theory (physical particles are neutrinos) are thus different from those in the theory of holes (physical particles are neutrino-holes and neutrinos with positive energy). In the ordinary theory, if we put

we obtain ${ }^{1}$

$$
L(\nu)=\int_{-\infty}^{+\infty} \gamma+(x) \gamma(\nu+\alpha) \mathrm{d} x,
$$

$$
L(\nu) L+(\mu)-L+(\mu) L(\nu)=0 .
$$

In the hole theory, we must introduce a new quantized wave function, $\uparrow(\alpha, \varepsilon)$, with $\alpha \geqslant 0, \varepsilon= \pm 1$, by means of the equations

$\varphi(\alpha, 1)=\gamma(\alpha) ; \varphi(\alpha,-1)=\gamma^{\dagger}(-\alpha) ;(\alpha \geqslant 0)$.

The operator (I) transforms ${ }^{4}$ into

$$
\begin{aligned}
K(\nu) & =\sum_{\varepsilon} \int_{0}^{\infty} \varphi+(\alpha, \varepsilon) \varepsilon \varphi(\alpha+\nu, \varepsilon) d \alpha+ \\
& +\int_{0}^{\nu} \varphi(\nu-\alpha,-1) \varphi(\alpha, 1) d x \quad(\nu \geqslant 0),
\end{aligned}
$$

and satisfies the Jordan-Kronig commutation rules

$$
K(v) K+(\mu)-K+(\mu) K(\nu)=\mu \delta(\mu-v) .
$$

Jordan and Kronig put $\sqrt{ } v b(v)=K(v)$. But the Bose amplitudes $b(v)$ are defined by their commutation rules only if $b(\nu)$ and $b+(\nu)$ form a complete set of operators, in the sense that any operator commuting with them must be a $c$-number. This can only be the case if the parameter $v$ in $b(v)$ includes all the degrees of freedom contained in the arguments of the quantized wave function $\varphi$ and of the corresponding wave functionals.

This is not the case in Jordan's theory, since $\varphi(\nu, \varepsilon)$ contains, besides $\nu$, a new degree of freedom $\varepsilon$, corresponding to a replacement of a neutrino by an antineutrino.

From this it follows that: (1) in Jordan's theory the operators $b(v)$ do not form a complete set and are not uniquely determined (we might take for example $\sqrt{ } \nu b(\nu)=K(\nu)-K(0)$ as well); and (2) all the probabilities and mean values referring to light quanta depend essentially on the values taken by the $\varepsilon$ variable.

But a neutrino cannot be distinguished by any physical means from an antineutrino, since according to the very neutrino theory their charge, their mass and their spin are the same and they possess no other physical characteristics. Thus the dependence of the above probabilities on the circumstance whether a particle is a neutrino $(\varepsilon=+1)$ or an antineutrino $(\varepsilon=-1)$ constitutes a very serious objection to the neutrino theory. If one remembers that the superposition principle is also violated in this theory and that only neutrinos having strictly parallel momentum - the probability of which is zero-are supposed to contribute to the formation of a light quantum, it is difficult to avoid the conclusion that a consistent neutrino theory of light is impossible.

Leningrad.

V. Fock.

I Fock, V., O.R. (Doklady). Acad. Sci. U.R.S.S., 4 (13), No. 5 (109), 229 (1936);' NATURE, 138, 1011 (1936).

2 Stueckelberg, E., NATURE, 139, 198 (193i).

3 Nath, Nagendra, Nature, 139, 331 (1937).

' ef. Sokolow, A., Nature, 139, 1071 (1937), where a similar formula is given.

\section{Number of Fragments of the Puttusk Meteorite}

IT is generally stated that the remarkable meteorite fall near Pułtusk, Poland, on January 30, 1868, consisted of 100,000 stones. Thus Charles P. Olivier in his monograph "Meteors"1 states that "the famous fall at Pultusk . . consisted of perhaps 100,000 fragments". Also F. Heide cites in his book ${ }^{2}$ the same number, and places this fall as the highest with regard to number of fragments (in the second place comes the shower at Holbrook, Arizona, on July 19, 1912, with 14,000 fragments). The same number is quoted by various works on astronomy and cosmography.

In 1936 I visited the Mineral Department of the British Museum (Natural History) in London, which contains a beautiful collection of fifty-nine Puttusk meteorites ; and in the note placed on the collection I found again the total number "estimated at 100,000 ". The same value is given also in the guide to the collection of meteorites by Dr. G. T. Prior ${ }^{3}$.

In spite of this remarkable agreement of data, the high number of 100,000 fragments does not seem to correspond with the facts. Indeed, in the original report on the Pultusk meteorite, published in 1868 by the University of Warsaw 4 , we find that only 400 fragments had been collected, although the total number of stones which reached the ground at the time was greater. On the other hand, the Polish "Cosmography" by J. Jedrzejewicz" estimates the amount of the Pultusk shower at about 1,000 stones, 